\title{
Quantifying Quantum Information via Uncertainties
}

\author{
Barış Öztop, Alexander A. Klyachko and Alexander S. Shumovsky \\ Faculty of Science, Bilkent University, Bilkent, \\ Ankara, 06800 Turkey \\ boztop@fen.bilkent.edu.tr
}

\begin{abstract}
We show, for a state $\psi$ of a quantum system with the dynamic symmetry given by the Lie group $G$, total amount of quantum information and entanglement is provided by summarized uncertainty of basic observables.

(C)2007 Optical Society of America

OCIS codes: $(000.6800)$ Theoretical physics, $(000.1600)$ Classical and quantum physics
\end{abstract}

For any given quantum system, it is necessary to know the minimal set of observables whose measurement in a state $\psi$ gives us the whole possible amount of quantum information carried by $\psi$. This is an important problem of quantum entanglement as well as of quantum information as a whole.

Within von Neumann theory of quantum measurements, all Hermitian observables are supposed to be equally accessible. As far as we know, Wick, Wightman and Wigner were the first who doubted this von Neumann's concept [1]. Later, Hermann [2] stated that definition of a quantum system should include specification of Lie algebra of allowed observables. In about the same time, Wigner [3] and Yanase [4] have defined the so-called skew information as a measure for the amount of information which an ensemble described by a state vector of a statistical matrix contains with respect to the macroscopically measured quantities. In the case of pure states, this measure simply coincides with the variance (uncertainty) of the observables (for further development of the concept of skew information, see [5]).

These ideas have been used recently to formulate an approach to quantum entanglement (see for review a [6] and references therein). In the present work, we discuss further development of this approach.

Consider a quantum system defined in the Hilbert space $H$ and having the dynamic symmetry given by the Lie group $G=\mathrm{SU}(H)$. We define the basic observables, specifying states of the system, as an orthogonal basis of the Lie algebra $L$ associated with the dynamic symmetry group: $G=\exp (i L)$.

Let us now note that the same space $H$ can be used to specify states of physical systems with different dynamic symmetry. For example, the three-dimensional Hilbert space, which is usually associated with description of qutrits, can be considered in the context of true qutrits with $G=\mathrm{SU}(3)$ [7] as well as in connection with spin-qutrits with reduced dynamic symmetry $G^{\prime}=\mathrm{SU}(2)$ [8]. The former system is characterized by eight basic observables (basis of the su(3) algebra. The latter one is defined by only three basic observables corresponding to the basis of the su(2) algebra. This circumstance causes a certain relativity of quantum information with respect to the dynamic symmetry of quantum system under consideration.

If the variance (uncertainty) of an observable $X_{j}$ is associated with the skew information [4], then the total variance

$$
V=\sum_{j}\left\langle X_{j}^{2}\right\rangle-\left\langle X_{j}\right\rangle^{2}
$$

gives the total amount of quantum information inherent in the measurement of all basic observables in a given state. The quantity (1) has the Casimir number $C=\sum_{j} X_{j}^{2}$ as the upper bond. Thus, the maximum of quantum information corresponds to the states in which

$$
\left\langle X_{j}\right\rangle=0 \quad \forall X_{j} \in \operatorname{basis}(L)
$$

In turn, the lower bond $V_{\min }$ can be associated with the generalized coherent states [9].

In particular case of two-qubit systems, it is possible to show one-to-one correspondence between (1) and Wootters' "entanglement of formation" that defines the specific "entanglement quantum information" [10]. The total variance (1) can be used for quantification of entanglement beyond bipartite systems as well [11]. 


\section{JWC59.pdf}

Besides that, the concept of quantum information expressed in terms of the quantity (1) has a number of logical advantages. In particular, it allows one to clarify the meaning of Bell's conditions in the theory of quantum entanglement [12] and to establish contact between quantum information and invariant theory in mathematics [13].

We propose to use the quantity (1) for quantifying quantum information carried by mixed states as well. In this case, an algorithm proposed in [14] can be used together with "purification" of quantum fluctuations by means of the action of operators from the complexified dynamic symmetry group $G^{c}=\exp (L \otimes C)$. This operation is usually associated with the stochastic local operation assisted by quantum communications (SLOCC) [15]. The approach can be easily illustrated in terms of multi-qubit and qutrit systems.

One of the authors (B. Ö.) would like to acknowledge the Scientific and Technical Research Council of Turkey (TÜBİTAK) for financial support.

\section{References}

[1] G.C. Wick, A.S. Wightman and E.P. Wigner, Phys. Rev. 88, 101 (1952).

[2] R. Hermann, Lie groups for physicists (Benjamin, New York, 1966).

[3] E.P. Wigner, Z. Physik 131, 101 (1952); Physikerlagung Wien (Physsik Verlag, Mosbach/Baden, 1962), p.1.

[4] E.P. Wigner and M.M. Yanase, Proc. Nat. Acad. Sci. USA 49, 910 (1963).

[5] F.J. Dyson and A. Leonard, J. Math. Phys. 8, 423 (1967); F.J. Dyson, J. Math. Phys. 8, 1538 (1967); E.H. Lieb and M.B. Ruskai, Phys. Rev. Lett. 30, 434 (1973); P. Gibilisco and T. Isola, J. Math. Phys. 44, 3752 (2003); S.-L. Luo and Q. Zhang, Phys. Rev. A 69, 032106 (2004).

[6] A.A. Klyachko and A.S. Shumovsky, J. Phys: Conf. Series 36, 87 (2006).

[7] C.M. Caves and G.J. Milburn, Opt. Commun. 179, 439 (2000).

[8] H. Barnum, E. Knill, G. Ortiz, R. Somma, and L. Viola, Phys. Rev. Lett. 92, 107902 (2004); M.A. Can, A.A. Klyachko, and A.S. Shumovsky, J. Opt. B: Quant. Semiclass. Opt. 7, L1 (2005).

[9] A.O. Barut and L. Girardello, Commun. Math. Phys. 21, 41 (1971); R, Gilmore, Lie groups, Lie algebras, and some of their applications (Wiley, New York, 1974); A.M. Perelomov, Generalized coherent states and their applications (Springer-Verlag, Berlin, 1986).

[10] W.K. Wootters, Phys. Rev. Lett. 80, 2245 (1998).

[11] A.A. Klyachko, B. Öztop and A.S. Shumovsky, Appl. Phys. Lett. 88, 124102 (2006); Phys. Rev. A (2007) (in print), quant-ph/0606119.

[12] A.A. Klyachko, J. Phys.: Conf. Series 36, 87 (2006).

[13] A.A. Klyachko, in Physics and Theoretical Computer Science, Edited by J.-P. Gazeau, J. Nešetril, and B. Rovan, Proc. of NATO-ASI on Physics and Theoretical Computer Science, Cargese, France, Octomer 17-29, 2005 (IOS Press, Amsterdam, 2007).

[14] F. Mintert, M. Kuś and A. Buchleitner, Phys. Rev. Lett. 92, 167902 (2004); F. Mintert, Ph.D. Thesis (2004), http://edoc.ub.unimuenchen.de/archive/00002133.

[15] W. Dür, G. Vidal, and J.I. Cirac, Phys. Rev. A 62, 062314 (2000); F. Verstraete, J. Dehaene, and B. De Moor, Phys. Rev. A 68, 012103 (2003). 